\title{
Quantitative nuclear histomorphometry predicts oncotype DX risk categories for early stage ER+ breast cancer
}

\author{
Jon Whitney ${ }^{1 *}$, German Corredor ${ }^{2}$, Andrew Janowczyk ${ }^{1}$, Shridar Ganesan ${ }^{3}$, Scott Doyle ${ }^{4}$, John Tomaszewski ${ }^{4}$,
} Michael Feldman ${ }^{5}$, Hannah Gilmore ${ }^{6}$ and Anant Madabhushi ${ }^{1}$

\begin{abstract}
Background: Gene-expression companion diagnostic tests, such as the Oncotype DX test, assess the risk of early stage Estrogen receptor (ER) positive (+) breast cancers, and guide clinicians in the decision of whether or not to use chemotherapy. However, these tests are typically expensive, time consuming, and tissue-destructive.

Methods: In this paper, we evaluate the ability of computer-extracted nuclear morphology features from routine hematoxylin and eosin (H\&E) stained images of 178 early stage ER+ breast cancer patients to predict corresponding risk categories derived using the Oncotype DX test. A total of 216 features corresponding to the nuclear shape and architecture categories from each of the pathologic images were extracted and four feature selection schemes: Ranksum, Principal Component Analysis with Variable Importance on Projection (PCA-VIP), Maximum-Relevance, Minimum Redundancy Mutual Information Difference (MRMR MID), and Maximum-Relevance, Minimum Redundancy - Mutual Information Quotient (MRMR MIQ), were employed to identify the most discriminating features. These features were employed to train 4 machine learning classifiers: Random Forest, Neural Network, Support Vector Machine, and Linear Discriminant Analysis, via 3-fold cross validation.

Results: The four sets of risk categories, and the top Area Under the receiver operating characteristic Curve (AUC) machine classifier performances were: 1) Low ODx and Low mBR grade vs. High ODx and High mBR grade (Low-Low vs. High-High) (AUC = 0.83), 2) Low ODx vs. High ODx (AUC = 0.72), 3) Low ODx vs. Intermediate and High ODx (AUC=0.58), and 4) Low and Intermediate ODx vs. High ODx (AUC $=0.65$ ). Trained models were tested independent validation set of 53 cases which comprised of Low and High ODx risk, and demonstrated per-patient accuracies ranging from 75 to 86\%.

Conclusion: Our results suggest that computerized image analysis of digitized H\&E pathology images of early stage ER+ breast cancer might be able predict the corresponding Oncotype DX risk categories.
\end{abstract}

\section{Background}

Estrogen Receptor positive (ER+) breast cancers are a common subtype of breast cancer that can frequently be effectively treated using hormonal therapy if deemed to have a low risk of recurrence. However, early stage ER+ breast cancers that are at high risk of recurrence are typically treated with adjuvant chemotherapy in addition to hormonal therapy. While chemotherapy increases survival rates by reducing rates of recurrence in these high risk subgroups [1], there may be significant side

\footnotetext{
* Correspondence: Jon.whitney@case.edu

${ }^{1}$ Department of Biomedical Engineering, Case Western Reserve University,

2071 Martin Luther King Drive, Cleveland, OH 44106-7207, USA

Full list of author information is available at the end of the article
}

effects including loss of hair, taste, cognitive function, and additional extensive medical care [2]. As such, it is critical to be able to determine the level of recurrence risk to plan treatment effectively so that the toxic side effects of chemotherapy can be avoided in low-risk patients.

Several methods of assessing tumor risk have been developed, including gene assays such as the Oncotype DX (ODx) Recurrence score, that stratify patients based on their risk of cancer recurrence [3]. The ODx test is a 21 gene assay that is currently employed for separating breast cancer patients into low and high risk of recurrence categories to help a clinician decide whether or not to prescribe adjuvant chemotherapy for early stage ER+ breast

(c) The Author(s). 2018 Open Access This article is distributed under the terms of the Creative Commons Attribution 4.0 International License (http://creativecommons.org/licenses/by/4.0/), which permits unrestricted use, distribution, and 
cancers [4]. The recurrence score is derived from the expression levels of multiple cancer-related genes, and ranges from 0 to 100 [4]. Patients with an ODx score of 17 or below are in the low-risk category, patients with ODx scores between 18 and 30 were considered intermediate risk, and scores 31 and above are in the high ODx risk category [5]. Unfortunately, Oncotype DX and similar companion diagnostic tests (e.g. Mammaprint [6], PAM50 [7]) tend to be expensive and time consuming due to the need for physical shipping of tissue samples to proprietary testing facilities. They are also tissue-destructive, making additional evaluation of other biomarkers or genes difficult.

The modified Bloom Richardson (mBR) grading scale is based on measuring nuclear grade (variation in nuclear shape and size), mitotic count, and tubule density. Each of these individual histologic primitives are assigned a score from 1 to 3 and then added to generate the cumulative mBR grade. Mina et al. [8] showed that mBR grade was also highly correlated the expression of proliferation genes used in the determination of ODx risk categories, and Flanagan et al. [9] identified a positive correlation between ODx risk category and nuclear grade when creating a predictive model of ODx based off clinical variables. Unfortunately, pathologic assessments of tumor grade are known to suffer from inter-observer variability [10].

Quantitative histomorphometry $(\mathrm{QH})$ refers to the use of computer-aided image analysis of digitized pathology images to "unlock" more revealing sub-visual attributes about tumor morphology, which can possibly be correlated with disease recurrence independent of other clinical and pathologic features. These features might also potentially reveal the underlying biology or molecular phenotype of the tumor. For example, Buchelli et al. showed that the number of mitoses identified via a deep learning algorithm was predictive of the ODx risk categories [11].

Nuclear architecture is another image attribute that has been implicated in the prediction of overall cancer grade and cancer aggressiveness [12, 13]. Additionally, variations in nuclear shape could reflect genetic instability [14] and may impact the ability of cancer cells to travel through tissue and create metastases that lead to recurrence [15]. A number of recent studies have shown the association of $\mathrm{QH}$ features of nuclear architecture and morphology with disease progression in oropharyngeal cancers [16], cancer recurrence in lung cancers [17], biochemical recurrence in prostate cancers $[18,19]$ and overall breast cancer survival [20].

There is also evidence that the performance of $\mathrm{QH}$ analysis improves when done separately on different cell types [20]. In the context of distinguishing breast cancers with different degrees of risk, it is likely that these cancers are characterized by different phenotypical changes in different cell types. Breast cancers are predominantly carcinomas -cancers which are derived from epithelial cells [21]. In addition, there is evidence that stromal cells react to tumor growth over time, and stromal phenotype can reflect a given cancer's genetic profile [22, 23]. For instance in [20], Beck et al. showed the importance of stromal morphology in predicting overall breast cancer survival. It is therefore useful to consider the behavior of epithelial and stromal cells as distinct groups when profiling breast cancer.

In this paper we evaluate the nuclear morphologic features to distinguish digitized images of H\&E sections from early stage ER+ breast cancers into ODx risk categories using supervised machine learning classifiers. ODx risk categories are comprised of three groups to reflect distinctions based off 5 year survival: low, intermediate, and high risk [5, 24]. However, there is both a high degree of correlation between ODx risk categories and $\mathrm{mBR}$ grade [8], as well as overlap between the intermediate and low and intermediate and high risk categories, making accurate separation of intermediate cases from other risk categories difficult [25]. We have therefore selected four categories to distinguish using computer extracted nuclear morphology features: 1) Low ODx and Low mBR grade vs. High ODx and High mBR grade (Low-Low vs. High-High) to evaluate whether nuclear morphology features were able to predict risk category when both the difficult to classify intermediate cases and differences between $\mathrm{mBR}$ grade and ODx risk category are removed. 2) Low ODx vs. High ODx to evaluate the predictive ability of the nuclear morphology features when difficult to classify intermediate cases are removed. 3) Low ODx vs. Intermediate and High ODx to evaluate the ability of the nuclear morphology features to identify the low ODx cases specifically. 4) Low and Intermediate ODx vs. High ODx to evaluate the ability of the nuclear morphology features to identify high ODx cases specifically.

The approach presented in this paper comprises the following main steps (Fig. 1). First, H\&E slides of surgical or biopsy specimens of breast tissue are scanned and digitized (Fig. 1.1). Second, nuclear segmentation is performed using deep learning models trained on manual breast nuclei annotations, followed by watershed separation to resolve overlapping nuclei (Fig. 1.2). Third, a deep learning model was used to separate epithelial from stromal regions, helping us identify which nuclei were stromal and which were epithelial (Fig. 1.3). Fourth, we extracted nuclear architectural and shape features from the epithelial and stromal regions separately (Fig. 1.4). Fifth, we perform feature selection on the resulting features using four different feature ranking schemes - Ranksum, PCA-VIP, MRMR MID, and MRMR MIQ. The predictive performance of these features was evaluated using four different supervised machine learning classifiers - random forest, support vector machine (SVM), linear discriminant analysis (LDA), and a neural network - via a 3-fold cross validation scheme (Fig. 1.5). The classifiers were 


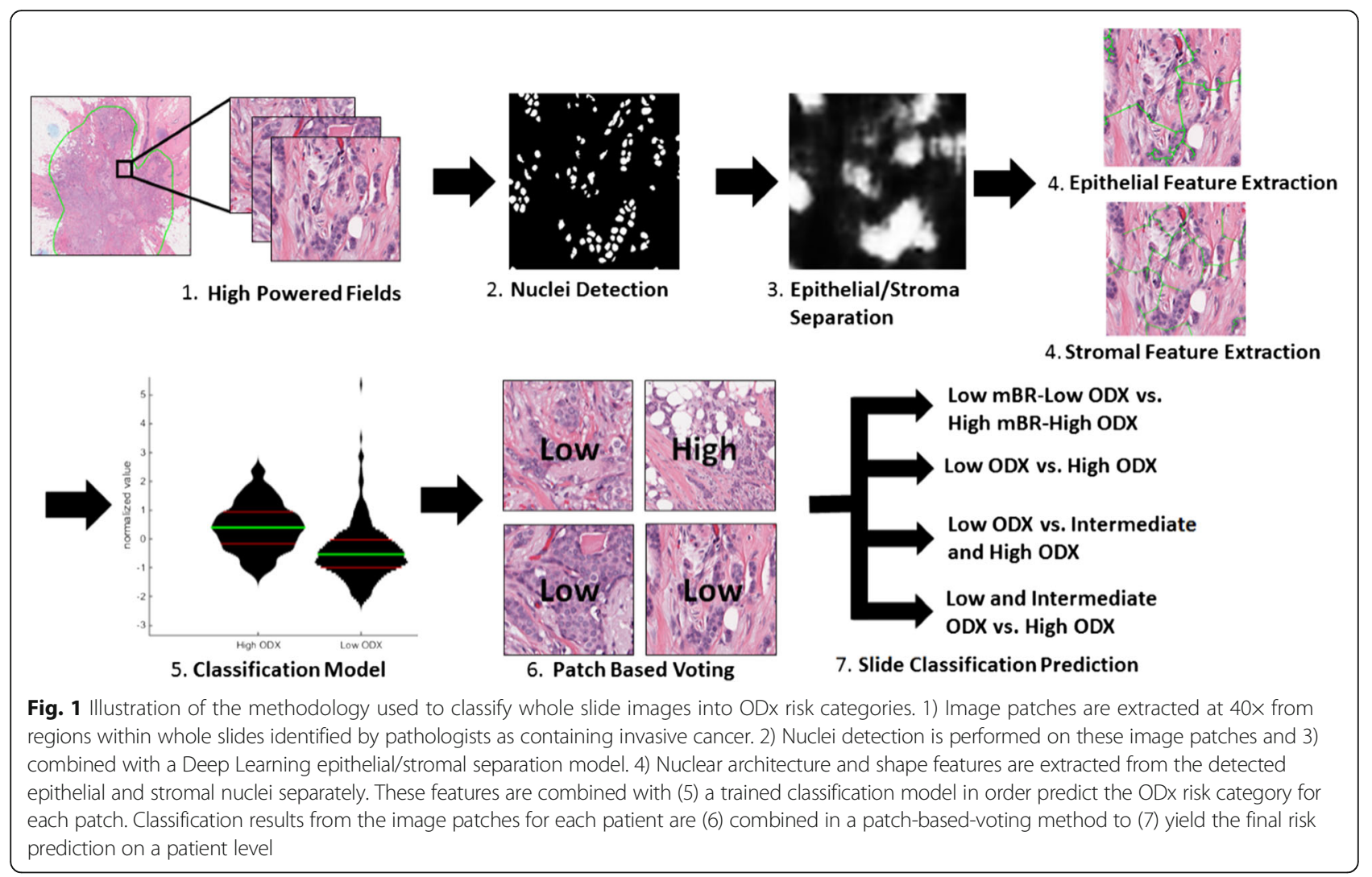

evaluated by their ability to distinguish between the four different classification tasks presented above using the area (AUC) under the Receiver Operating Characteristic (ROC) curve, which plots the true positive rate against the false positive rate. Finally, classifiers are trained to create per-patch risk category predictions, identifying the optimal threshold of what percentage of positively classified patches should result in a positive prediction based on training data, and then applied and evaluated on testing folds to create a final prediction of the ODx risk category for each patient (Fig. 1.6, 1.7).

\section{Methods}

\section{Dataset description}

Our study comprised of $178 \mathrm{H} \& \mathrm{E}$ stained whole tissue slides of ER+ Lymph node negative breast cancer patients (Table 1). These whole slide breast cancer samples dataset was selected to include 1) early stage ER+ breast cancers, 2) surgically resected tissue specimens, and 3) the availability of a corresponding Oncotype DX risk score. These slides were obtained from patients treated between 2004 and 2009 at the Cancer Institute of New Jersey and the University of Pennsylvania, and between 2008 and 2013 at Case Western Reserve University. Slides were locally digitized at their originating institutions using Aperio, Leica, and Philips scanners. The Modified Bloom-Richardson Grade for each of the pathologic specimens was determined by pathologists at each of the participating institutions. 9 cases in which the mBR score and ODx risk category were at opposite extremes (4 low mBR and High ODx, and 5 high $\mathrm{mBR}$ and low ODx) were excluded from this study.

\section{Nuclei segmentation}

We employed the approach described in [26] by Janowczyk et al. for segmenting individual nuclei. Two Deep Learning (DL) models were employed. The first model identified the likelihood that a given pixel was part of a nucleus and the second model identified the likelihood that a pixel was part of the epithelium or stroma. Both models were trained using manual segmentations of the tissue primitives of interest (i.e. nucleus or stroma or epithelium). DL was executed using Caffe, a popular open-source DL framework [27]. The DL models were trained using $32 \times 32$ sized image patches on a Titan XGPU running CUDA 7.5, and a 9-layer convolutional neural network framework.

The nuclear segmentation model was trained on a dataset of 141 manually annotated ER+ breast cancer tissue images, each patch sized $2000 \times 2000$ pixels and at $40 \times$ magnification. The epithelium/stroma separation model was trained on a dataset of $236 \mathrm{ER}+$ breast cancer tissue image patches, each sized at $1000 \times 1000$ pixels and at $10 \times$ magnification. Lower magnification in the epithelial/stromal separation model allowed for more contextual information to be included in the image patches 
Table 1 Dataset characteristics - demographic and cancer subtype distribution in each risk category for the cases from the 3 different institutions considered in this study

\begin{tabular}{|c|c|c|c|}
\hline \multicolumn{4}{|l|}{ Parameters } \\
\hline Oncotype DX Risk Category & Low $(<18)$ & Intermediate $(>18, \leq 30)$ & $\operatorname{High}(>30)$ \\
\hline No. of Patients $(N=125)$ & $66(53 \%)$ & $44(35 \%)$ & $15(12 \%)$ \\
\hline Age & $20-77$ & $25-70$ & $45-70$ \\
\hline \multicolumn{4}{|l|}{ Sex } \\
\hline Female & $66(53 \%)$ & $43(34 \%)$ & $15(12 \%)$ \\
\hline Male & $0(0 \%)$ & $1(1 \%)$ & $0(0 \%)$ \\
\hline \multicolumn{4}{|l|}{ Patient Ethnicity } \\
\hline White & $33(26 \%)$ & $23(18 \%)$ & $5(5 \%)$ \\
\hline African American & $3(2 \%)$ & $2(2 \%)$ & $2(2 \%)$ \\
\hline Asian & $1(1 \%)$ & $2(2 \%)$ & $1(1 \%)$ \\
\hline Unknown & $22(18 \%)$ & $17(14 \%)$ & $7(6 \%)$ \\
\hline \multicolumn{4}{|l|}{ PR Status } \\
\hline Positive & $64(51 \%)$ & 39 (31\%) & $10(8 \%)$ \\
\hline Negative & $2(2 \%)$ & $3(2 \%)$ & $5(4 \%)$ \\
\hline Unknown & $0(0 \%)$ & $2(2 \%)$ & $0(0 \%)$ \\
\hline \multicolumn{4}{|l|}{ HER2 Status } \\
\hline Positive & $0(0 \%)$ & $1(1 \%)$ & $0(0 \%)$ \\
\hline Negative & $66(53 \%)$ & $42(34 \%)$ & $15(12 \%)$ \\
\hline Unknown & $0(0 \%)$ & $1(1 \%)$ & $0(0 \%)$ \\
\hline \multicolumn{4}{|l|}{ Histologic Tumor Grade } \\
\hline $\operatorname{Low}(4,5)$ & $10(8 \%)$ & $14(11 \%)$ & $0(0 \%)$ \\
\hline Moderate $(6,7)$ & $48(38 \%)$ & $24(19 \%)$ & $4(3 \%)$ \\
\hline High $(8,9)$ & $8(6 \%)$ & $6(5 \%)$ & $11(9 \%)$ \\
\hline \multicolumn{4}{|l|}{ Tumor Type } \\
\hline Ductal & $53(42 \%)$ & $37(30 \%)$ & $14(11 \%)$ \\
\hline Ductal With Lobular Features & $9(7 \%)$ & $3(2 \%)$ & $1(1 \%)$ \\
\hline Ductal with Mucinous Features & $1(1 \%)$ & $2(2 \%)$ & $0(0 \%)$ \\
\hline Mixed & $3(2 \%)$ & $2(2 \%)$ & $0(0 \%)$ \\
\hline
\end{tabular}

during model training, improving accuracy and speed. This patch-based approach allowed for multiple identicallysized image patches to be used, increasing the size of the training set. In addition, the patch size was selected to use the field of view identified as being optimal for extracting nuclear architecture features of the tumor [28].

\section{Feature extraction}

A total of 216 nuclear features were extracted from epithelial and stromal nuclei separately, resulting in a total of 432 features per patch. These features consisted of architecture and shape features.

Architectural features were obtained by performing quantitative analysis of nuclear graphs, such as Delaunay Triangles, Voronoi Diagrams, Minimum Spanning Trees (MST), and Cell Cluster Graphs (CCG) [29] (Fig. 2). These nuclear graphs were constructed using the individual nuclei as the vertices of the graph. The choice of vertex connectivity determines the type of nuclear graph (i.e. Delaunay, Voronoi, MST, CCG) constructed. Features extracted from the graphs included changes in the lengths of edges and distance between nearest vertices. Cellular disorder can be measured using features derived from Cell Orientation Graphs [19]. Shape features included Invariant Moment, Fourier Descriptor, and Length/Width ratios. A comprehensive enumeration of all the image features extracted is presented in the Additional file 1.

\section{Feature ranking}

Feature ranking was used to identify the most relevant image features for predicting the corresponding ODx risk category. Features were ranked in order of highest relevance to the classification problem. The most relevant features identified were subsequently used in conjunction 


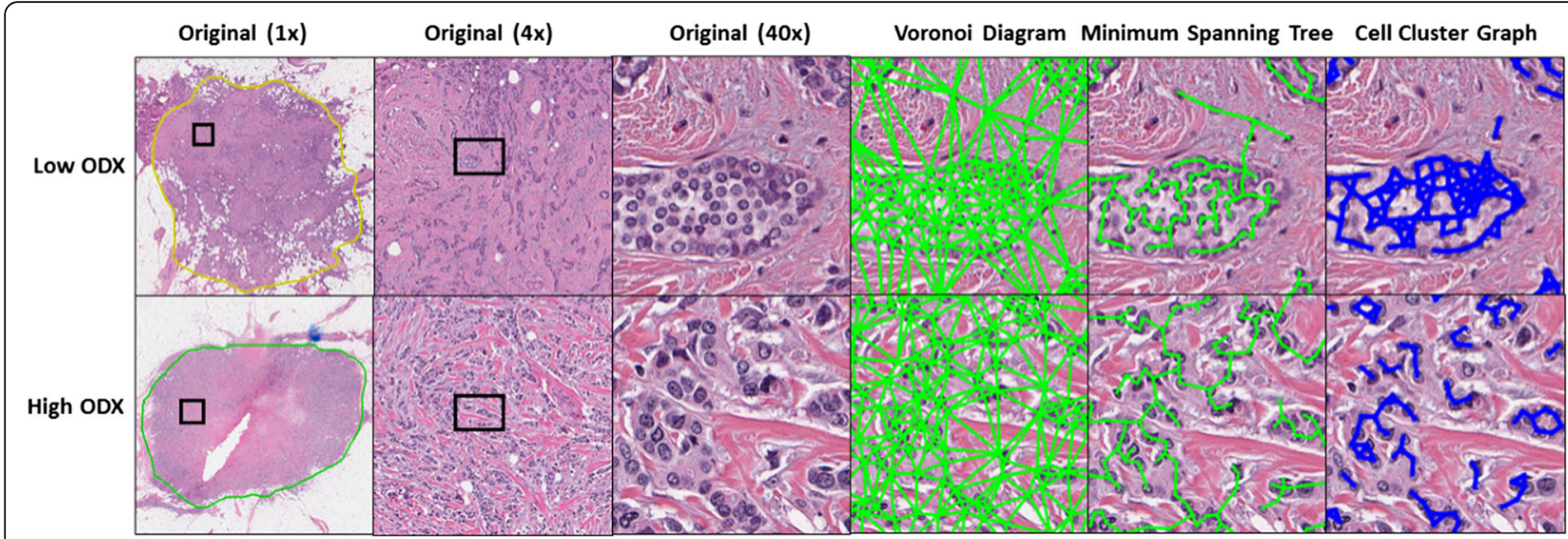

Fig. 2 Nuclear graphs used to calculate features relating to spatial arrangement of nuclei. Left to right: Original images at $1 \times, 4 \times$, and $40 \times$, Voronoi Diagram, Minimum Spanning Tree, and Cell Cluster Graph, reflecting local nuclear architecture. Comparison between graph appearance for a low ODx example (top) and a high ODx example (bottom)

with machine learning classifiers. A number of popular feature ranking methods were evaluated including Wilcoxon Ranksum [30], PCA-VIP [31], and Maximum-Relevance Minimum-Redundancy (MRMR) [32] with two variants Mutual Information Difference, and Mutual Information Quotient (MRMR-MID and MRMR-MIQ) [33]. Each of these feature ranking methods takes a slightly different approach to identifying the most relevant features, and simultaneously suppressing features that are highly correlated with each other. The Ranksum method identifies feature relevance to classification without explicitly considering the correlation between highly-ranked features [30]. PCA-VIP uses a combination criteria of both how each of the principle component vectors relate to the outcome to be predicted, and which features most highly contribute to those principle component vectors (effectively measuring to what extent a given feature provides unique information in a dataset) [31]. MRMR-MID and MRMR-MIQ both use maximal relevance criteria which use the mean mutual information values between features and the relevant output class, while minimizing the redundancy (mutual information between any feature and the other features in the dataset) [32].

\section{Classifier construction}

A total of four different classifiers was tested in conjunction with each of the four different feature selection methods. The classifiers employed included a bagged C4.5 Random Forest [34], a ten-node four-layer Neural Network [35], a 3 kernel Support Vector Machine [36], and a pseudolinear discriminant Linear Discriminant Analysis [37]. Machine learning classifiers were trained using 100 iterations of randomly initialized 3-fold cross-validation. 3-fold crossvalidation was employed to divide the entire dataset of image patches into three equal groups by patient ID, thus ensuring that patches from each patient were not simultaneously present in the training and hold-out groups. Two of these groups were used for model training, while the third group was used to test the trained model. Machine learning classifiers were trained on a perpatch basis. This allowed for a simple patch-based voting method, in which the classification of the patient as being in the low or high-risk category was based on if the number of class labels predicted for a given class surpassed a patch percentage threshold. The optimal threshold was determined from the training data in each iteration. This method can also be used to classify individual patches spatially in an H\&E slide, providing a spatially distributed assessment of cancer aggression across a given sample (Fig. 3).

\section{Experiments \\ The four experiments were as follows}

1) Low ODx and Low mBR grade vs. High ODx and High mBR grade (Low-Low vs. High-High). This experiment was used to look at the cases reflecting the extremes in terms of tumor morphology and ODx risk. While grade and ODx risk scores are correlated for the most part [8], in this experiment we chose to ignore conflicting cases (i.e. cases with a low $\mathrm{mBR}$ grade but a high ODx score and vice-versa).

2) Low ODx vs. High ODx. This experiment looks at cases of high distinction in terms of ODx risk category, but does not exclude cases with conflicting grade categories.

3) Low ODx vs. Intermediate and High ODx. This is the hypothesis that is closest to the question a clinician is interested in answering: identifying cases that are low ODx risk score from all others so that low ODx risk patients can avoid aggressive chemotherapies. 


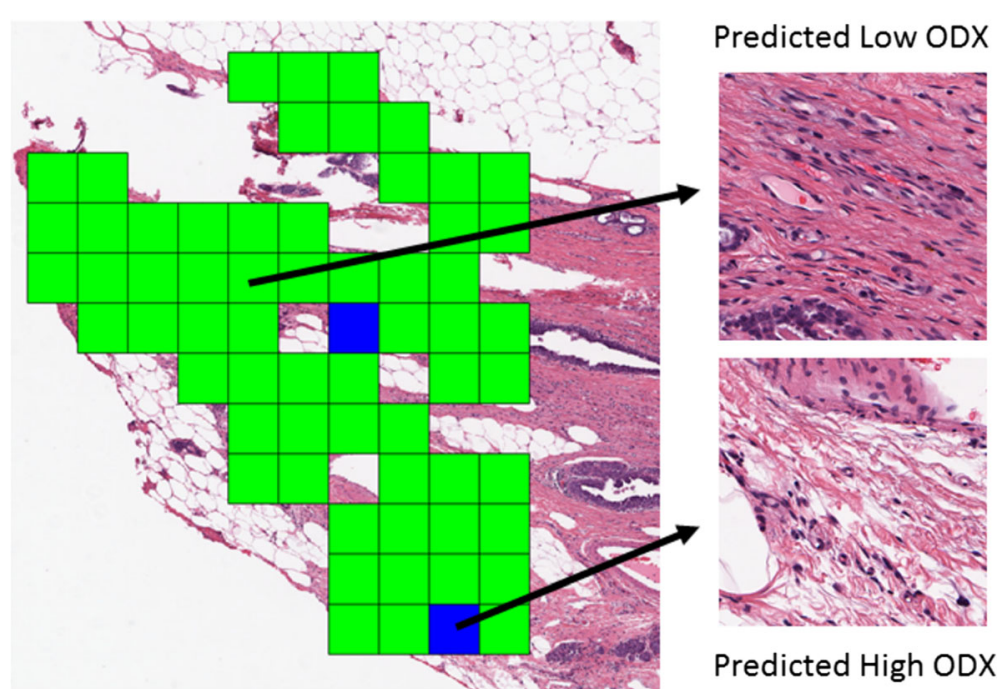

Fig. 3 Example of the Low-Low vs. High-High random forest classifier using ranksum feature selection applied to patches from whole slide image. Machine classification uses the top ranked epithelial and stromal features. Green squares indicate patches that are predicted to be Low ODx while Blue squares are predicted to be High ODx

4) Low and Intermediate ODx vs. High ODx. This experiment considers the possibility that high ODx risk patients are histologically distinct from both other ODx risk categories.

We also quantitatively assessed the performance of each of four different feature ranking methods over stromal and epithelial features in conjunction with four different machine learning classification schemes to determine which combination of classification and feature ranking approaches resulted in the highest per-patient patch voting accuracy for each of the four experiments. Per-patient patch voting simply means that the classifier was applied to each patch extracted from a patient, thus generating an ODx risk category prediction for each patch. A simple majority of the per-patch risk category predictions for each patient is then used to determine the predicted patient ODx risk category. The per-patient patch voting accuracy is defined as the percentage of patients whose ODx risk category was correctly predicted using this method.

\section{Feature evaluation via supervised classification}

For each of the 4 classification experiments described above, we identified 1) the most highly ranked and predictive epithelial and stromal nuclear morphologic features which were evaluated via violin plots (Figs. 4), and 2) classification accuracy for the machine learning classifiers in conjunction with the top ranked features in the form of AUC.

Violin plots illustrate the distribution of normalized feature values for the top performing features between the two risk categories. Thus, high degrees of separation between the two distributions indicate a high level of discrimination from that feature. AUC curves indicate the true positive rate as a function of the false positive rate at varying confidence thresholds. The higher the area under the curve (indicated by the curve extending into the upper left quadrant), the more frequently the classifier is able to correctly identify the class, and the less frequently it is to falsely classify a case as positive. For comparison, a diagonal line extending from the bottom left to the upper right corner would indicate an AUC of 0.5, which is considered to be the equivalent of guessing.

In order to demonstrate the significance of epithelial/ stromal separation, we ran two sets of features using the optimized machine learning classifier and feature ranking algorithm. The two feature sets were: 1) nuclei features extracted from all nuclei, 2) nuclei features extracted from epithelial and stromal nuclei separately. The utility of separating epithelial and stromal nuclei prior to feature extraction was measured by comparing the AUCs between models trained from features with no epithelial/stromal separation, and epithelial stromal separation prior to feature extraction.

\section{Evaluation of models on external validation set}

In order to fully assess the effectiveness of the models generated, the models with the highest performance were used on an external validation set. Models were trained over the entire primary cohort before being applied without any retraining to the external validation set.

\section{Results}

The results for the four primary experiments are as follows

1) Low ODx and Low mBR grade vs. High ODx and High mBR grade (Low-Low vs. High-High) (Fig. 5, 


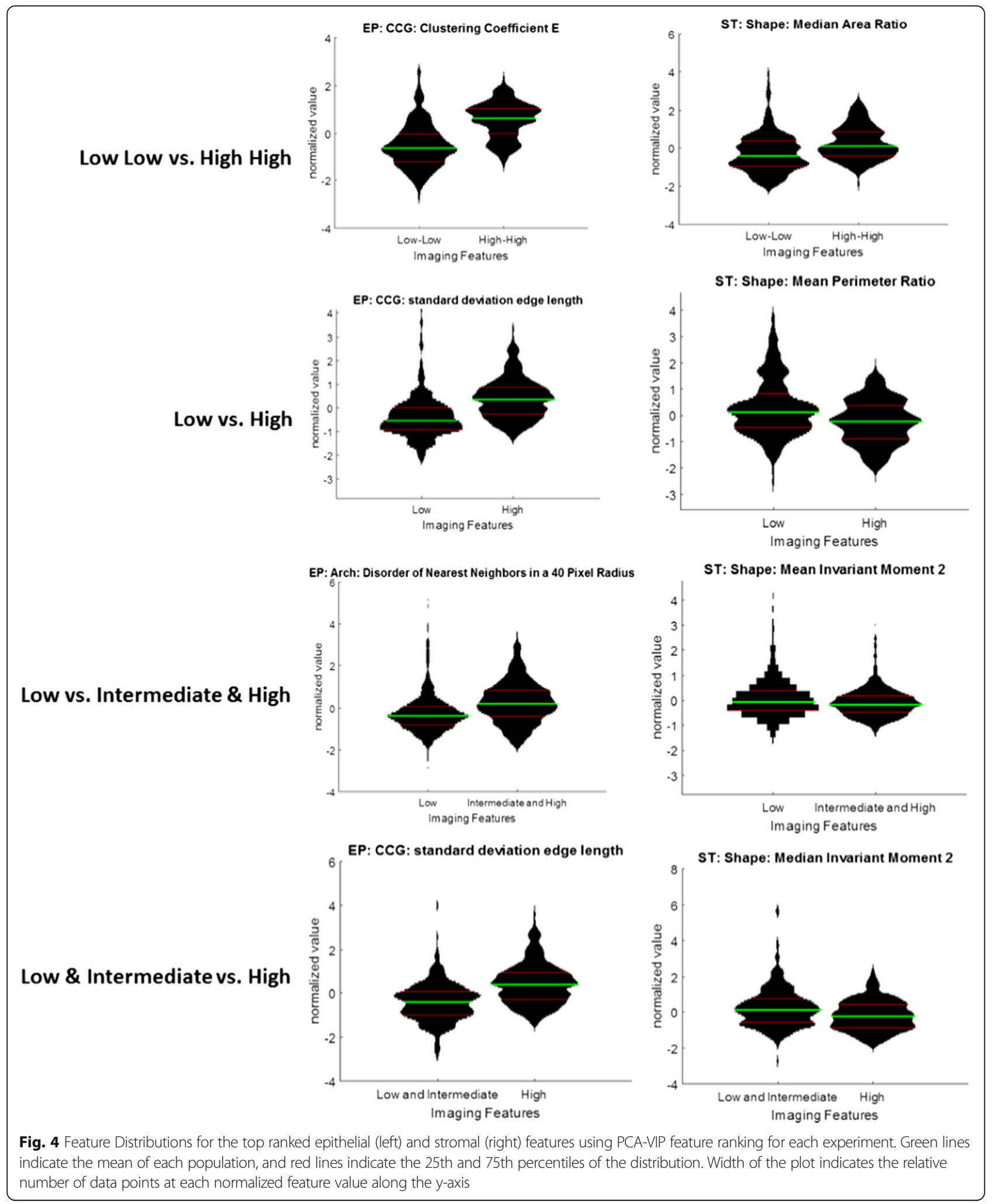

top left). In this experiment, the top ranked epithelial features were cell cluster graphs, and the top ranked stromal features were shape features related to nuclear perimeter, area ratios, and invariant moment (Table 3). The SVM classifier using the PCA-VIP feature ranking scheme yielded the highest classification accuracy with 
Low Low vs. High High

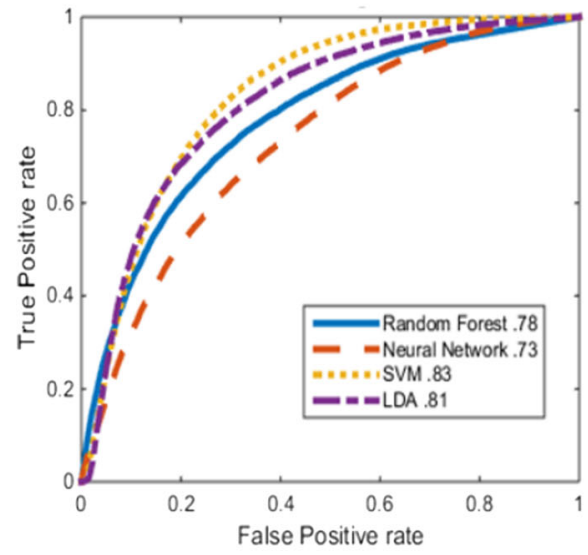

Low vs. Intermediate \& High

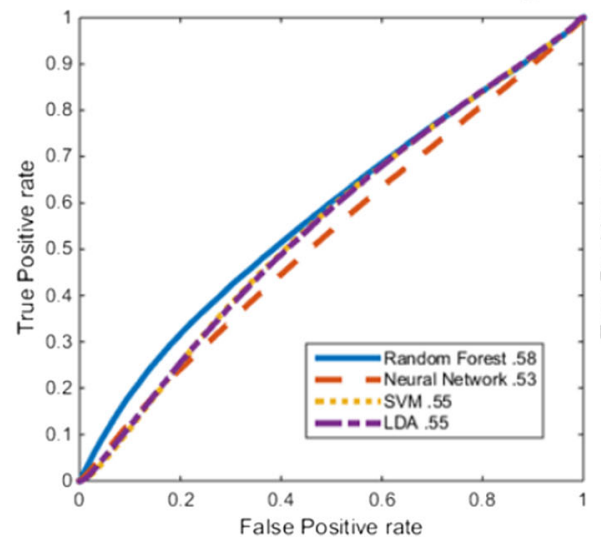

Low vs. High

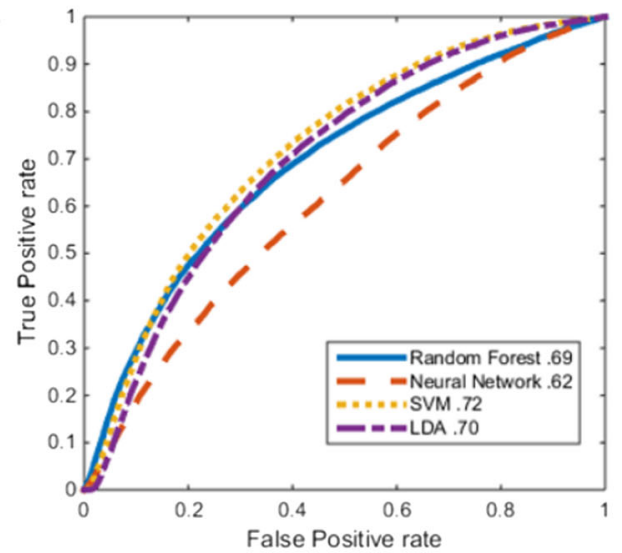

Low \& Intermediate vs. High

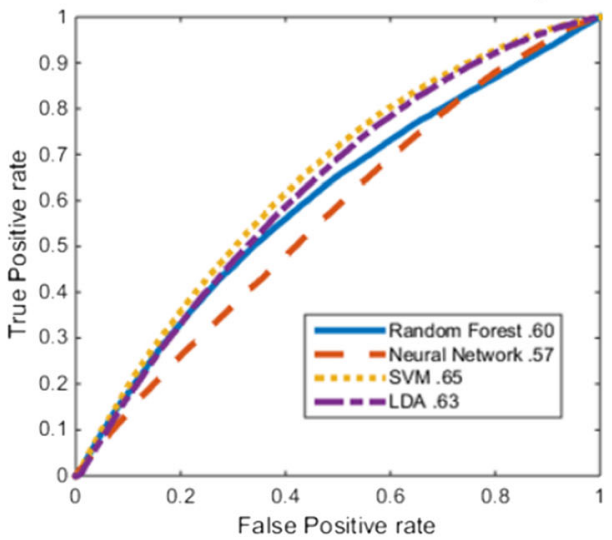

Fig. 5 ROC curves for each of the four experiments conducted (panels) and classification methods (lines) using PCA-VIP feature selection. Top left: Low ODx and Low mBR grade vs. High ODx and High mBR grade (Low-Low vs. High-High). Top Right: Low ODx vs. High ODx. Bottom Left: Low ODx vs. Intermediate and High ODx. Bottom Right: Low and Intermediate ODx vs. High ODx. Each panel displays the ROC curve using either (solid) random forest, (dashed) neural network, (dotted) SVM, or (intermediate dash) LDA classification. Feature set includes epithelial and stromal features. AUC values for each curve are displayed in the legend

an AUC of 0.83 , and a patch voting accuracy of $86 \%$ (Table 2). AUC results using the same classifier and feature ranking methodology improved from 0.71 to 0.83 with the inclusion of stromal features (Table 4).

2) Low ODx vs. High ODx (Fig. 5, top right) (Fig. 5, top right): The top ranked epithelial features were the cell cluster graph and disorder of nearest neighbors features, while the highest ranked stromal features were similar to those identified for the low-low vs. high-high discrimination problem, namely perimeter ratio, area ratio, and invariant moment (Table 3). The SVM classifier using the PCA-VIP feature ranking scheme yielded a classification AUC of 0.72 , and a patch voting accuracy of $76 \%$ (Table 2). AUC results using the same classifier and feature ranking methodology improved from 0.61 to 0.72 with the separation of epithelial and stromal nuclei (Table 4).
3) Low ODx vs. Intermediate and High ODx (Fig. 5, bottom left): The top ranked epithelial features were primarily disorder and number of nearest neighbors features, while the highest ranked stromal features were primarily metrics regarding the invariant moment (Table 3). The random forest classifier using the PCA-VIP feature ranking scheme yielded a classification AUC of 0.58, and a patch voting accuracy of $64 \%$ (Table 2). AUC results using the same classifier and feature ranking methodology improved from 0.55 to 0.58 with the separation of epithelial and stromal nuclei (Table 4).

4) Low and Intermediate ODx vs. High ODx (Fig. 5, bottom right):: The top ranked epithelial features were metrics concerning the mean and variation in edge length associated with cell cluster graphs, while the highest ranked stromal features were the invariant moment and standard deviation of the Fourier descriptor (Table 3). The SVM classifier 
Table 2 Classification accuracy metrics for each of the four experiments. From left to right: Low ODx Low mBR vs. High ODx Low mBR, Low ODx vs. High ODx, Low ODx vs. Intermediate and High ODx, and Low and Intermediate ODx vs. High ODx. Data for each experiment includes the AUC, best patch Voting Accuracy results, and the optimal feature ranking and classifier used to achieve the optimized patch voting accuracy results. All experiments conducted with 3-fold cross-validation

\begin{tabular}{lllll}
\hline Experiment & LL vs. HH & L vs. H & L vs. Int. and H & L and Int. vs. H \\
\hline Number of Patients & 37 & 75 & 125 & 111 \\
AUC & 0.81 & 0.69 & 0.58 & 0.6 \\
AUC STDev & 0.08 & 0.05 & 0.03 & 0.06 \\
Patch Voting Accuracy & $82 \%$ & $80 \%$ & $60 \%$ & $86 \%$ \\
Best Feat. Ranking for Patch voting & MRMR-MID & PCA-VIP & Ranksum & MRMR-MID \\
Best Classifier for Patch voting & LDA & Random Forest & Random Forest & Random Forest \\
\hline
\end{tabular}

and PCA-VIP feature ranking scheme yielded an AUC of 0.65 , and a patch voting accuracy of $74 \%$ (Table 2). AUC results using the same classifier and feature ranking methodology improved from 0.55 to 0.65 with the separation of epithelial and stromal nuclei (Table 4).
Of the epithelial features considered, the most discriminating features identified across all 4 classification problems were those pertaining to epithelial architecture of nuclei (Table 3). Of the stromal features, the most significant tended to be those related to measuring changes in the shape of the stromal nuclei. In each experiment, the

Table 3 Top three Epithelial and Stromal features for each of the four experiments: Low ODx and Low mBR grade vs. High ODx and High mBR grade (Low-Low vs. High-High), Low ODx vs. High ODx, Low ODx vs. Intermediate and High ODx, and Low and Intermediate ODx vs. High ODx

\begin{tabular}{|c|c|c|}
\hline Experiments & Epithelial Features (EP) & \\
\hline \multirow[t]{3}{*}{ Low Low vs. High High } & 1 & EP: CCG: Clustering Coefficient E \\
\hline & 2 & EP: CCG: standard deviation edge length \\
\hline & 3 & EP: CCG: Clustering Coefficient D \\
\hline \multirow[t]{3}{*}{ Low vs. High } & 1 & EP: CCG: standard deviation edge length \\
\hline & 2 & EP: CCG: mean edge length \\
\hline & 3 & EP: Arch: Disorder of Nearest Neighbors in a 40 Pixel Radius \\
\hline \multirow[t]{3}{*}{ Low vs. Intermediate and High } & 1 & EP: Arch: Disorder of Nearest Neighbors in a 40 Pixel Radius \\
\hline & 2 & EP: Arch: Disorder of Nearest Neighbors in a 50 Pixel Radius \\
\hline & 3 & EP: Arch: Avg. Nearest Neighbors in a 40 Pixel Radius \\
\hline \multirow[t]{4}{*}{ Low and Intermediate vs. High } & 1 & EP: CCG: standard deviation edge length \\
\hline & 2 & EP: CCG: mean edge length \\
\hline & 3 & EP: Arch: Disorder of Nearest Neighbors in a 40 Pixel Radius \\
\hline & Stromal Features (ST) & \\
\hline \multirow[t]{3}{*}{ Low Low vs. High High } & 1 & ST: Shape: Median Area Ratio \\
\hline & 2 & ST: Shape: Median Invariant Moment 2 \\
\hline & 3 & ST: Shape: Mean Perimeter Ratio \\
\hline \multirow[t]{3}{*}{ Low vs. High } & 1 & ST: Shape: Mean Perimeter Ratio \\
\hline & 2 & ST: Shape: Mean Area Ratio \\
\hline & 3 & ST: Shape: Mean Invariant Moment 2 \\
\hline \multirow[t]{3}{*}{ Low vs. Intermediate and High } & 1 & ST: Shape: Mean Invariant Moment 2 \\
\hline & 2 & ST: Shape: Median Invariant Moment 2 \\
\hline & 3 & ST: Shape: Standard Deviation Invariant Moment 2 \\
\hline \multirow[t]{3}{*}{ Low and Intermediate vs. High } & 1 & ST: Shape: Median Invariant Moment 2 \\
\hline & 2 & ST: Shape: Mean Invariant Moment 2 \\
\hline & 3 & ST: Shape: Standard Deviation Fourier Descriptor 2 \\
\hline
\end{tabular}


Table 4 Improvements in classification accuracy based on features extracted from all nuclei together (No Ep/St. Sep.) vs. features extracted from epithelial nuclei and stromal nuclei separately (Ep/St Sep.), ranked via the PCA-VIP feature selection scheme, and used to train an SVM classifier. All AUC scores were generated using 3-fold cross validation

\begin{tabular}{llll}
\hline Experiment & No Ep/St Separation & Ep/St Separation & AUC Improvement \\
\hline High-High vs. Low-Low & 0.71 & 0.83 & 0.12 \\
High vs. Low & 0.61 & 0.72 & 0.11 \\
Low vs. Intermediate and High & 0.55 & 0.58 & 0.03 \\
Low and Intermediate vs. High & 0.55 & 0.65 & 0.1 \\
Average & 0.61 & 0.7 & 0.09 \\
\hline
\end{tabular}

epithelial features were identified to be more significant in separating the different risk categories compared to the stromal nuclei features (Fig. 6). The classification AUC for the machine learning classifier was highest for the problems involving the extreme risk or grade categories (i.e. Low-Low vs High-High and Low ODx vs High ODx). Unsurprisingly, the AUC values were lower when the intermediate risk category was also included (i.e. Low ODx vs. Intermediate and High ODx and Low and Intermediate ODx vs. High ODx).
In addition, while each of the feature ranking methods had very comparable performance, the PCA-VIP feature ranking scheme yielded slightly better performance, with a peak AUC of 0.71 using a Support Vector Machine (Fig. 6).

Comparisons between the classification efficacy with and without the use of epithelial/stromal separation across the four experiments yielded an average improvement of 0.09 (Table 4).
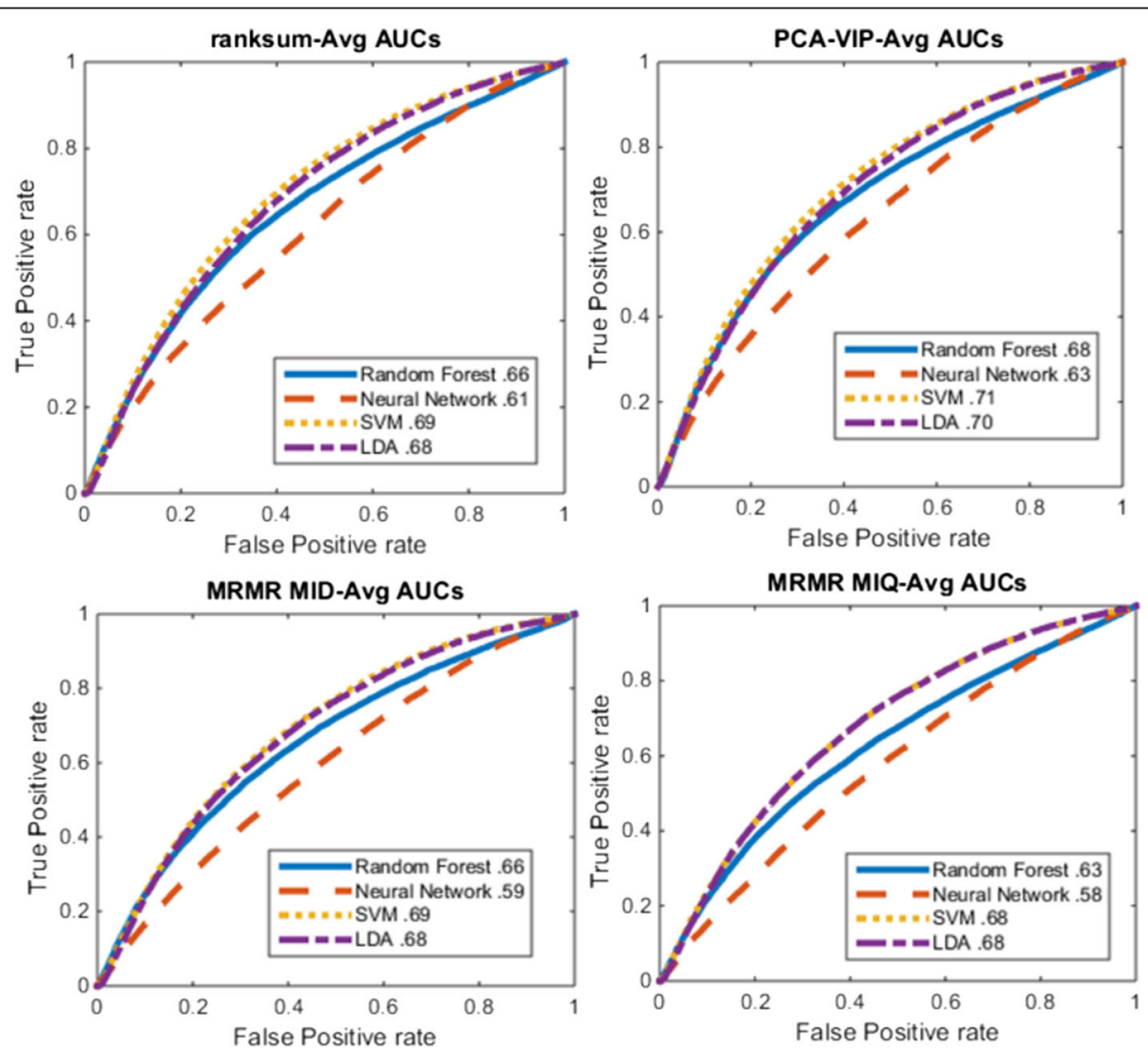

Fig. 6 Determining the optimal feature ranking method - ROC curves for different combinations of feature ranking methods (panels) and classification methods (lines) for separating low from high ODx patches. Top left: Ranksum (Wilcoxon rank sum). Top right: PCA-VIP. Bottom left: MRMR-MID. Bottom right: MRMR-MIQ. Each panel displays the ROC curve using either (solid) random forest, (dashed) neural network, (dotted) SVM, or (intermediate dash) LDA classification. Feature set includes stromal and epithelial features. AUC values for each curve are displayed in the legend 


\section{Validation results}

We tested the results of the model on an external validation set. The model was trained using Ranksum feature ranking and a Random forest classifier using 100 iterations of 3-fold cross-validation to determine the top-performing features. These features were then trained over the entire training set before being evaluated on the validation set. The validation set was obtained from the University of Pennsylvania and contained 53 cases comprised of Low and High ODx risk cases of primarily Low and High mBR grade (Table 5). As described previously, the accuracy of each model was determined using per-patient patch voting, where pathologist selected ROIs were divided into sub-ROI patches, and each patch was then classified as belonging to either low or high risk using each of the four models. The classification of the patient into high or low risk was determined by the percentage of sample patches predicted to belong to either category. Because it is possible that the optimal percentage threshold for distinguishing between high and low risk may not be a simple majority, the ideal percentage of patches that were need to be identified as low for the patient to be categorized as low ODx risk was determined from the training set. Perpatient accuracies ranged between 76 and 85\% across all hypotheses evaluated. Improvements in classification accuracy of low vs. high over low-low vs high-high may be explained by the fact that the validation set was composed exclusively of low and high ODx samples. In addition, the larger number of samples which were low ODx as compared to high ODx samples may explain why the model trained to distinguish between low and intermediate vs high had slightly improved performance over the model trained to distinguish between low vs. intermediate and high. It may also reflect the fact that the low and intermediate risk patients are more alike from a histomorphometric perspective compared to the intermediate and high risk patients. The accuracies were highest using models trained to distinguish between Low vs. High and Low vs (Intermediate and High ODx) cases (Table 6).

\section{Discussion}

In this work, we evaluated the effectiveness of computerextracted measurements of size, shape, and architectural features of epithelial and stromal nuclei in separating early

Table 5 Validation dataset characteristics - ODx and grade distribution

\begin{tabular}{llll}
\hline $\begin{array}{l}\text { Validation Set }(N=53) \\
\text { mBR Tumor Grade\ODx } \\
\text { Category }\end{array}$ & Low $(<18)$ & $\begin{array}{l}\text { Intermediate } \\
(>18, \text { leq30) }\end{array}$ & High $(>30)$ \\
Low $(4,5)$ & $40(75 \%)$ & $0(0 \%)$ & $0(0 \%)$ \\
Moderate $(6,7)$ & $0(0 \%)$ & $0(0 \%)$ & $1(2 \%)$ \\
High $(8,9)$ & $0(0 \%)$ & $0(0 \%)$ & $12(23 \%)$ \\
\hline
\end{tabular}

Table 6 Validation dataset - Classification accuracy using Ranksum feature ranking and a SVM classifier for each of four classification separations

Ranksum - SVM \& Classification Accuracy

Low-Low vs. High-High

Low vs. High

$79 \%$

Low and Intermediate vs. High

$85 \%$

Low vs. Intermediate and High

$84 \%$

stage ER+ breast cancer histology samples into different Oncotype DX determined risk categories. Nuclear feature extraction was accomplished by 1) obtaining nuclear segmentations with a deep learning algorithm, 2) using deep learning epithelial/stromal separation of nuclei, and 3) extracting nuclei shape and architectural features from those segmentations. Those features were then given to a series of machine based classifiers and feature ranking methods using 3-fold cross-validation to test the effectiveness of each machine based classifier. These features were then employed in the context of discriminating the following 4 different grade-ODx risk categories: 1) Low ODx and Low mBR grade vs. High ODx and High mBR grade (Low-Low vs. High-High). 2) Low ODx vs. High ODx. 3) Low ODx vs. Intermediate and High ODx. 4) Low and Intermediate ODx vs. High ODx.

We found that the best classifier accuracy $(\mathrm{AUC}=0.83$ ) was obtained for the Low-Low vs. High-High classification problem. Since the ODx risk category is strongly correlated with tumor grade [9], by choosing to leave out conflicting cases (i.e. where the grade and ODx risk categories are not aligned), the Low-Low vs High-High categories represent the extreme risk cases. The next highest accuracy was obtained for the Low ODx vs. High ODx categories, where all intermediate risk cases were left out. The best classifier AUC obtained in this experiment (AUC $=0.72$ ) was lower compared to the AUC obtained for the Low-Low vs HighHigh problem, possibly due to presence of 64 cases (55 Intermediate $\mathrm{mBR}$ and Low $\mathrm{ODx}$, and 9 Intermediate mBR High ODx) where the grade and ODx risk categories did not align. This most likely adversely affected the training and the evaluation of the machine learning classifiers. When evaluating the classifiers in distinguishing the Low vs. Intermediate and High and the Low and Intermediate vs. High ODx risk categories, the Low and Intermediate vs. High ODx distinction had slightly improved performance as compared to distinguishing Low vs. Intermediate and High ODx risk categories. This may be due to the fact that the intermediate cases identified by ODx were primarily low risk cases [38].

Classifier models trained on Low vs. High and the Low with Intermediate vs. High ODx cases yielded the highest classification accuracy on the validation set. These results appear to suggest that histomorphometrically the low 
ODx and intermediate ODx appeared more similar compared to the high ODx cases. Clearly this will need to be validated in additional, larger independent validation studies, but if confirmed might suggest that a number of the patients currently classified as intermediate risk by Oncotype DX might actually be low risk and should be classified as such.

Tumor grade is determined by tubule formation, nuclear pleomorphism, and mitotic count [39]. These same features are found to strongly correlate breast cancer outcome [40]. The state of tubule formation is reflected in features such as the ratio of tubule nuclei to total nuclei [41]. The architecture of tubule formation is also reflected in features used in the presented work, such as Cell Cluster Graphs [29], Cell Orientation Entropy [19], and Disorder of Nearest Neighbors [19]. Nuclear pleomorphism may be reflected in features such as the Mean Invariant Moment [42], and Area Ratio [43]. Thus, the features used in this work are implicitly reflective of the histomorphometric measurements used by pathologists to assess grade and breast cancer outcome. However, the method presented can also identify complex and sub-visual (i.e. information which is present, but not easily discernable by a human, such as higher-order nuclei architectural characteristics, or difficult to recognize chromatin patterns $[44,45])$ relationships between quantitative features and ODx categories that are difficult for pathologists to visually identify. The Oncotype gene expression test aims to capture changes in genetic expression in genes that have been tied with specific cancer-related traits [46]. For example, Ki-67, STK15, Survivin, Cyclin B1, and MYBL2 have all been associated with breast cancer proliferation; Stromelysin 3 and Cathepsin L2 have been associated with invasion; and $\mathrm{ER}, \mathrm{PR}, \mathrm{Bcl} 2$, and SCUBE2 have been associated with responsiveness to Estrogen [47]. Variations in these genes could potentially lead to changes in visual presentation of the cancer, and thus affect the features previously described. For example, increases in Ki-67 activity resulting in increased unregulated cell proliferation may increase the density of cell nuclei, resulting in an increase in the Disorder of Nearest Neighbors, or decreased distance between nuclei in Cell Cluster Graphs. Tumor invasion resulting from activation of Stromelysin 3 could result in either a loss of tissue differentiation, or the presence of large epithelial nuclei invading into the surrounding stroma [48]. These types of phenotypic changes might be captured by architectural features, or size and shape variation amongst stromal nuclei features. For example, variation in stromal nuclei shape could also be related to the connection between spindle-cell and round stromal nuclei contact and breast cancer patient survival discovered by Beck et al. [20].

Previous groups have been able to duplicate ODx results using equations drawing from genetic expression and pathologist grading information, such as the Magee Equation [9]. Using these methods, low grade and low ER and PR $(\leq 150)$ can be correctly categorized as being low ODx $89 \%$ of the time; and when ignoring intermediate ODx cases, low and high ODx samples can be correctly identified with concordance rates between 96.9 and 100\% $[25,49]$. However, these methods have between 54.3 and $59.4 \%$ concordance when considering intermediate cases as well as low and high, and require pathologist-generated data [25]. When considering the intermediate risk categories, our classification AUC ranged from 0.58 and 0.6 which appears to be in alignment with the findings in [25].

Several different groups have previously explored the use of $\mathrm{QH}$ for predicting ODx risk categories. For example, Basavanhally et al. was able to separate high from low grade breast cancer patients, with top performing architectural features such as Delaunay Triangle metrics, nuclei density, and Voronoi Diagram architectural information [12]. Romo-Bucheli et al. was able to separate high-high from low-low cases with an AUC of 0.76 using a single feature: the ratio of tubule nuclei to non-tubule nuclei [41]. This approach used Deep Learning to identify biologically relevant structures (separating tubule nuclei from nontubule nuclei), while the presented approach used a much larger number of nuclei-specific features for classification purposes.

While related to these previous approaches [12], our focus was on quantitatively evaluating the role of computer extracted features of nuclear morphology in the stroma and epithelium with the Oncotype Dx risk categories. Additionally, unlike previous related studies [13] our study looked at the most discriminating features to distinguish not just the extreme risk categories (low vs. high) but also looked at the ability of computer extracted nuclear morphologic features to distinguish the intermediate risk categories from the low and high risk categories.

We do however acknowledge the several limitations of this work. Firstly, the validation set used only included high and low ODx cases, without any intermediate cases. Secondly, the focus of this work was on finding features that were associated with ODx risk categories and not patient outcome. Oncotype DX is a companion diagnostic test, and while the risk categories have been validated against outcome, it is not perfectly correlated [50]. Unfortunately, long-term disease recurrence or patient outcome information was not available for the cases considered in this study. We also did not conduct a detailed study of the influence of staining and scanning variations on the features identified as predictive and the influence of these parameters on the subsequent classification results. Finally, we focused solely on the role of nuclear morphology in this work, there are clearly other features that are known to have a prognostic role in early stage ER+ breast 
cancers, features relating to number and distribution of tumor infiltrating lymphocytes, mitoses [11], and tubules [41]. These features have shown to be independently useful in determining ODx risk categories in ER+ breast cancer, and would likely improve the classification results when combined with the nuclear histomorphometric features presented in this work. Another potential future avenue is the integration of histomorphometric approaches such as this with genomic based tests to determine if the integration of morphologic and molecular measurements enables more accurate risk assessment, especially for the patients currently identified as intermediate risk. We hope to address these limitations in future work.

\section{Conclusions}

In this work we evaluated the role of computer extracted features relating to spatial architecture and shape within the epithelium and stroma and showed that these features could distinguish early stage ER+ breast cancers into different ODx risk categories. Our results suggest that with additional validation, these features could be used to create an inexpensive, rapid, and nondestructive predictor of low and high ODx risk categories for early stage ER+ breast cancer based off digitized images of H\&E slides alone.

\section{Additional file}

Additional file 1: Table S7. Features tested for significance, and considered for use in final analysis. Comprehensive list of features investigated for classification utility. Each feature was used to analyze epithelial and stromal nuclei separately. (XLSX 15 kb)

\section{Abbreviations \\ CCG: Cell Cluster Graph; ER +: Estrogen Receptor Positive; H\&E: Hematoxylin and eosin; LDA: Linear Discriminant Analysis; mBR: Modified Bloom-Richardson; MRMR MID: Maximum Relevance, Minimum Redundancy, Mutual Information Difference; MRMR MIQ: Maximum Relevance, Minimum Redundancy, Mutual Information Quotient; MST: Minimum Spanning Trees; ODx: Oncotype Dx; PCA-VIP: Primary Component Analysis - Variable Importance; QH: Quantitative Histomorphometry; ROC: Region Under the Curve; SVM: Support Vector Machine}

\section{Acknowledgements}

NVIDIA -a Titan X GPU, Gift of Titan X GPU to support research. Special thanks to Natalie Shih for helping procure validation data in a timely manner.

\section{Funding}

The following funding bodies provided funding for the data collection, digitization, annotation and the computational and statistical analysis, as also in the writing of the manuscript.

Research reported in this publication was supported by the National Cancer Institute of the National Institutes of Health under award numbers.

1U24CA199374-01,

R01CA202752-01A1.

R01CA208236-01A1.

R21CA179327-01;

R21CA195152-01.

The National Institute of Diabetes and Digestive and Kidney Diseases under award number R01DK098503-02,
National Center for Research Resources under award number 1 C06 RR12463-01.

The DOD Prostate Cancer Synergistic Idea Development Award (PC120857);

The DOD Lung Cancer Idea Development New Investigator Award

(LC130463),

The DOD Prostate Cancer Idea Development Award:

The DOD Peer Reviewed Cancer Research Program W81XWH-16-1-0329.

The Ohio Third Frontier Technology Validation Fund.

The Hartwell Foundation.

the Wallace H. Coulter Foundation Program in the Department of Biomedical Engineering and the Clinical and Translational Science Award Program (CTSA) at Case Western Reserve University.

The content is solely the responsibility of the authors and does not necessarily represent the official views of the National Institutes of Health.

\section{Availability of data and materials}

The datasets used and/or analyzed during the current study are available from the corresponding author on reasonable request.

\section{Authors' contributions}

All authors have read and approved the manuscript. JW, GC, and AJ were responsible for experiments run. Manuscript was written primarily by JW and AM, with support from all authors. SG, SD, JT, MF, and HG were responsible for defining the clinical problem, reviewing and annotating imaging data, and providing biological interpretation of the findings. Validation dataset provided by MF

Ethics approval and consent to participate

The study was HIPAA compliant and was approved by the Institutional Review Board at the University Hospitals Case Medical Center. The informed consent was waived by the institutional review board for this retrospective study.

\section{Competing interests}

Dr. Madabhushi is an equity holder in Elucid Bioimaging and in Inspirata Inc. $\mathrm{He}$ is also a scientific advisory consultant for Inspirata Inc. In addition, he currently serves as a scientific advisory board member for Inspirata Inc. and for Astrazeneca. He also has sponsored research agreements with Philips and Inspirata Inc. His technology has been licensed to Elucid Bioimaging and Inspirata Inc. He is also involved in a NIH U24 grant with PathCore Inc. and a R01 with Inspirata Inc. Drs John Tomaszewski. Michael Feldman and Shridar Ganesan are members of the scientific advisory board of Inspirata, Inc. a digital pathology start-up company, and receives board fees and stock options. The authors declare that they have no competing interests.

\section{Publisher's Note}

Springer Nature remains neutral with regard to jurisdictional claims in published maps and institutional affiliations.

\section{Author details}

${ }^{1}$ Department of Biomedical Engineering, Case Western Reserve University, 2071 Martin Luther King Drive, Cleveland, OH 44106-7207, USA. '2Universidad Nacional de Colombia, Bogotá D.C, Colombia. ${ }^{3}$ Department of Medicine, Division of Medical Oncology, Rutgers Robert Wood Johnson Medical School, Rutgers Cancer Institute of New Jersey, 195 Little Albany Street, New Brunswick, NJ 08903, USA. ${ }^{\text {SUNY }}$ at the University at Buffalo, 3435 Main Street, Buffalo, NY, USA. ${ }^{5}$ Department of Pathology, University of Pennsylvania Perelman School of Medicine, Philadelphia, PA, USA. ${ }^{6}$ Department of Pathology, University Hospitals, Cleveland Medical Center and Case Western Reserve University, Cleveland, OH 44106, USA.

Received: 27 October 2017 Accepted: 26 April 2018

Published online: 30 May 2018

\section{References}

1. Early Breast Cancer Trialists' Collaborative Group (EBCTCG). Effects of chemotherapy and hormonal therapy for early breast cancer on recurrence and 15-year survival: an overview of the randomised trials. Lancet Lond Engl. 2005:365(9472):1687-717. https://doi.org/10.1016/S01406736(05)66544-0. PMID: 15894097 
2. Brezden CB, Phillips K-A, Abdolell M, Bunston T, Tannock IF. Cognitive function in breast cancer patients receiving adjuvant chemotherapy. J Clin Oncol. 2000;18(14):2695-701.

3. Paik S, Shak S, Tang G, Kim C, Baker J, Cronin M, Baehner FL, Walker MG, Watson D, Park T, Hiller W, Fisher ER, Wickerham DL, Bryant J, Wolmark N. A multigene assay to predict recurrence of tamoxifen-treated, node-negative breast cancer. N Engl J Med. 2004;351(27):2817-26. https://doi.org/10.1056/ NEJMoa041588. PMID: 15591335

4. Paik S, Tang G, Shak S, Kim C, Baker J, Kim W, Cronin M, Baehner FL, Watson D, Bryant J, Costantino JP, Geyer CE, Wickerham DL, Wolmark N. Gene expression and benefit of chemotherapy in women with node-negative estrogen receptor-positive breast Cancer. J Clin Oncol. 2006;24(23):3726-34. https://doi.org/10.1200/JCO.2005.04.7985.

5. Sparano JA, Gray RJ, Makower DF, Pritchard KI, Albain KS, Hayes DF, Geyer CE, Dees EC, Perez EA, Olson JA, Zujewski J, Lively T, Badve SS, Saphner TJ, Wagner LI, Whelan TJ, Ellis MJ, Paik S, Wood WC, Ravdin P, Keane MM, Gomez Moreno HL, Reddy PS, Goggins TF, Mayer IA, Brufsky AM, Toppmeyer DL, Kaklamani VG, Atkins JN, Berenberg JL, Sledge GW. Prospective validation of a 21-gene expression assay in breast Cancer. N Engl J Med. 2015;373(21):2005-14. https://doi.org/10.1056/NEJMoa1510764.

6. Wittner BS, Sgroi DC, Ryan PD, Bruinsma TJ, Glas AM, Male A, Dahiya S, Habin K, Bernards R, Haber DA, Van't Veer LJ, Ramaswamy S. Analysis of the MammaPrint breast Cancer assay in a predominantly postmenopausal cohort. Clin Cancer Res. 2008;14(10):2988-93. https://doi.org/10.1158/10780432.CCR-07-4723

7. Nielsen TO, Parker JS, Leung S, Voduc D, Ebbert M, Vickery T, Davies SR, Snider J, Stijleman IJ, Reed J, Cheang MCU, Mardis ER, Perou CM, Bernard PS, Ellis MJ. A comparison of PAM50 intrinsic subtyping with immunohistochemistry and clinical prognostic factors in tamoxifen-treated estrogen receptor-positive breast Cancer. Clin Cancer Res. 2010;16(21):522232. https://doi.org/10.1158/1078-0432.CCR-10-1282.

8. Mina L, Soule SE, Badve S, Baehner FL, Baker J, Cronin M, Watson D, Liu M-L, Sledge GW, Shak S, Miller KD. Predicting response to primary chemotherapy: gene expression profiling of paraffin-embedded core biopsy tissue. Breast Cancer Res Treat. 2007;103(2):197-208. https://doi.org/10.1007/ s10549-006-9366-x

9. Flanagan MB, Dabbs DJ, Brufsky AM, Beriwal S, Bhargava R. Histopathologic variables predict Oncotype DX recurrence score. Mod Pathol Off J U S Can Acad Pathol Inc. 2008;21(10):1255-61. https://doi.org/10.1038/modpathol. 2008.54. PMID: 18360352

10. Allsbrook WC, Mangold KA, Johnson MH, Lane RB, Lane CG, Epstein J. Interobserver reproducibility of Gleason grading of prostatic carcinoma: general pathologist. Hum Pathol. 2001;32(1):81-8. https://doi.org/10.1053/ hupa.2001.21135. PMID: 11172299

11. Romo-Bucheli D, Janowczyk A, Gilmore H, Romero E, Madabhushi A. A deep learning based strategy for identifying and associating mitotic activity with gene expression derived risk categories in estrogen receptor positive breast cancers. Cytom Part J Int Soc Anal Cytol. 2017; https://doi.org/10.1002/cyto. a.23065. PMID: 28192639

12. Basavanhally A, Ganesan S, Feldman M, Shih N, Mies C, Tomaszewski J, Madabhushi A. Multi-field-of-view framework for distinguishing tumor grade in ER+ breast cancer from entire histopathology slides. IEEE Trans Biomed Eng. 2013;60(8):2089-99. https://doi.org/10.1109/TBME.2013.2245129. PMID: 23392336

13. Basavanhally A, Feldman M, Shih N, Mies C, Tomaszewski J, Ganesan S, Madabhushi A. Multi-field-of-view strategy for image-based outcome prediction of multi-parametric estrogen receptor-positive breast cancer histopathology: comparison to Oncotype DX. J Pathol Inform. 2011;2:S1. https://doi.org/10.4103/2153-3539.92027. PMID: 22811953 PMCID: PMC3312707

14. Gisselsson D, Björk J, Höglund M, Mertens F, Dal Cin P, Åkerman M, Mandahl N. Abnormal nuclear shape in solid tumors reflects mitotic instability. Am J Pathol. 2001 Jan;158(1):199-206. https://doi.org/10.1016/ S0002-9440(10)63958-2

15. Trepat X, Wasserman MR, Angelini TE, Millet E, Weitz DA, Butler JP, Fredberg JJ. Physical forces during collective cell migration. Nat Phys. 2009;5(6):426-30. https://doi.org/10.1038/nphys1269.

16. Lewis JS, Ali S, Luo J, Thorstad WL, Madabhushi A. A quantitative histomorphometric classifier (QuHb|C) identifies aggressive versus indolent p16-positive oropharyngeal squamous cell carcinoma. Am J Surg Pathol. 2014;38(1):128-37. https://doi.org/10.1097/PAS.0000000000000086. PMID: 24145650 PMCID: PMC3865861
17. Yu K-H, Zhang C, Berry GJ, Altman RB, Ré C, Rubin DL, Snyder M. Predicting non-small cell lung cancer prognosis by fully automatede microscopic pathology image features. Nat Commun. 2016;7:12474. https://doi.org/10. 1038/ncomms12474.

18. Lee G, Veltri RW, Zhu G, Ali S, Epstein Jl, Madabhushi A. Nuclear shape and architecture in benign fields predict biochemical recurrence in prostate Cancer patients following radical prostatectomy: preliminary findings. Eur Urol Focus. 2016; https://doi.org/10.1016/j.euf.2016.05.009.

19. Lee G, Ali S, Veltri R, Epstein Jl, Christudass C, Madabhushi A. Cell orientation entropy (COrE): predicting biochemical recurrence from prostate cancer tissue microarrays. Med Image Comput Comput-Assist Interv MICCAI Int Conf Med Image Comput Comput-Assist Interv. 2013;16(Pt 3):396-403. PMID: 24505786

20. Beck AH, Sangoi AR, Leung S, Marinelli RJ, Nielsen TO, van de Vijver MJ, West RB, van de Rijn M, Koller D. Systematic analysis of breast cancer morphology uncovers stromal features associated with survival. Sci Transl Med. 2011;3(108):108ra113. https://doi.org/10.1126/scitrans/med.3002564. PMID: 22072638

21. American Cancer Society. Types of breast Cancer. [cited 2016 Aug 16]. Available from: http://www.cancer.org/cancer/breastcancer/detailedguide/ breast-cancer-breast-cancer-types

22. Bhowmick NA, Neilson EG, Moses HL. Stromal fibroblasts in cancer initiation and progression. Nature. 2004;432(7015):332-7. https://doi.org/10.1038/ nature03096.

23. Van den Eynden GG, Colpaert CG, Couvelard A, Pezzella F, Dirix LY, Vermeulen PB, Van Marck EA, Hasebe T. A fibrotic focus is a prognostic factor and a surrogate marker for hypoxia and (lymph)angiogenesis in breast cancer: review of the literature and proposal on the criteria of evaluation. Histopathology. 2007;51(4):440-51. https://doi.org/10.1111/j. 1365-2559.2007.02761.x. PMID: 17593207

24. Henson DE, Ries L, Freedman LS, Carriaga M. Relationship among outcome, stage of disease, and histologic grade for 22,616 cases of breast cancer. The basis for a prognostic index. Cancer. 1991;68(10):2142-9. https://doi.org/10. 1002/1097-0142(19911115)68:10<2142::AID-CNCR2820681010>3.0.CO;2-D.

25. Klein ME, Dabbs DJ, Shuai Y, Brufsky AM, Jankowitz R, Puhalla SL, Bhargava R. Prediction of the Oncotype DX recurrence score: use of pathology-generated equations derived by linear regression analysis. Mod Pathol. 2013;26(5):658-64. https://doi.org/10.1038/modpathol.2013.36.

26. Janowczyk A, Madabhushi A. Deep learning for digital pathology image analysis: A comprehensive tutorial with selected use case. J Pathol Inform. 2016.

27. Jia Y, Shelhamer E, Donahue J, Karayev S, Long J, Girshick R, Guadarrama S, Darrell T. Caffe: convolutional architecture for fast feature embedding. ACM Press; 2014 [cited 2016 Aug 4]. p. 675-678. Available from: http://dl.acm.org/ citation.cfm?doid=2647868.2654889. https://doi.org/10.1145/2647868. 2654889.

28. Basavanhally A, Ganesan S, Shih N, Mies C, Feldman M, Tomaszewski J, Madabhushi A. A boosted classifier for integrating multiple fields of view: breast cancer grading in histopathology: IEEE; 2011 [cited 2016 Aug 1]. p. 125-128. Available from: http://ieeexplore.ieee.org/lpdocs/epic03/wrapper. htm?arnumber=5872370. https://doi.org/10.1109/ISBI.2011.5872370.

29. Ali S, Veltri R, Epstein JA, Christudass C, Madabhushi A. Gurcan MN, Madabhushi A, editors. Cell cluster graph for prediction of biochemical recurrence in prostate cancer patients from tissue microarrays; 2013 [cited 2016 Mar 18]. p. $86760 \mathrm{H}$. Available from: http://proceedings. spiedigitallibrary.org/proceeding.aspx?doi=10.1117/12.2008695. https://doi. org/10.1117/12.2008695

30. Devore J. Probability and statistics for engineering and the sciences: Cengage Learning; 2015

31. Ginsburg SB, Viswanath SE, Bloch BN, Rofsky NM, Genega EM, Lenkinski RE, Madabhushi A. Novel PCA-VIP scheme for ranking MRI protocols and identifying computer-extracted MRI measurements associated with central gland and peripheral zone prostate tumors. J Magn Reson Imaging JMRI. 2015;41(5):1383-1393. https://doi.org/10.1002/jmri.24676. PMID: 24943647

32. Peng $H$, Long $F$, Ding $C$. Feature selection based on mutual information: criteria of max-dependency, max-relevance, and min-redundancy. IEEE Trans Pattern Anal Mach Intell. 2005;27(8):1226-1238. https://doi.org/10.1109/ TPAMI.2005.159 PMID: 16119262.

33. Ding $\mathrm{C}$, Peng $\mathrm{H}$. Minimum redundancy feature selection from microarray gene expression data. J Bioinforma Comput Biol. 2005;03(02):185-205. https://doi.org/10.1142/S0219720005001004. 
34. Strobl C, Malley J, Tutz G. An introduction to recursive partitioning: rationale, application, and characteristics of classification and regression trees, bagging, and random forests. Psychol Methods. 2009 ;14(4):323-348. https:// doi.org/10.1037/a0016973 PMID: 19968396 PMCID: PMC2927982.

35. Demuth H, Beale M. Neural network toolbox for use with Matlab - User's guide version. 1993.

36. Pelchmans K, Suykens J, Gestel T, Brabanter J, Lukaas L, Hamers B, Moor B, Vandewalle J. LS-SVMlab: a matlab/c toolbox for least squares support vector machines. 2002.

37. Izenman AJ. Linear discriminant analysis. In: Izenman AJ, editor. Mod Multivar stat tech Regres Classif manifold learn. New York, NY: Springer New York; 2008. p. 237-80. Available from: https://doi.org/10.1007/978-0-38778189-1_8.

38. JMS B, Bayani J, Marshall A, Dunn JA, Campbell A, Cunningham C, Sobol MS, Hall PS, Poole CJ, Cameron DA, Earl HM, Rea DW, Macpherson IR, Canney P, Francis A, McCabe C, Pinder SE, Hughes-Davies L, Makris A, Stein $\mathrm{RC}$, on behalf of the OPTIMA TMG. Comparing breast Cancer multiparameter tests in the OPTIMA prelim trial: no test is more equal than the others. J Natl Cancer Inst. 2016;108(9):djw050. https://doi.org/10.1093/ jnci/djw050.

39. Elston CW, Ellis IO. Pathological prognostic factors in breast cancer. I. The value of histological grade in breast cancer: experience from a large study with long-term follow-up. Histopathology. 1991 Nov;19(5):403-10. https:// doi.org/10.1111/j.1365-2559.1991.tb00229.x.

40. Bloom H, Richardson W. Histological grading and prognosis in breast Cancer: a study of 1409 cases of which 359 have been followed for 15 years. Br J Cancer. 1957:359-77.

41. Romo-Bucheli D, Janowczyk A, Romero E, Gilmore H, Madabhushi A. Automated tubule nuclei quantification and correlation with oncotype DX risk categories in ER+ breast cancer whole slide images. In: Gurcan MN, Madabhushi A, editors. 2016 [cited 2016 Aug 3]. p. 979106 . Available from: http://proceedings.spiedigitallibrary.org/proceeding.aspx?doi=10.1117/12. 2211368 doi:https://doi.org/10.1117/12.2211368

42. Ongun G, Halici U, Leblebicioglu K, Atalay V, Beksac M, Beksac S. Feature extraction and classification of blood cells for an automated differential blood count system: IEEE; 2001 [cited 2017 Jan 10]. p. 2461-2466. Available from: http://ieeexplore.ieee.org/document/938753/. https://doi.org/10.1109/ IJCNN.2001.938753.

43. Liotta LA, Kleinerman J, Saidel GM. Quantitative relationships of intravascular tumor cells, tumor vessels, and pulmonary metastases following tumor implantation. Cancer Res. 1974 May 1;34(5):997.

44. Madabhushi A. Computerized histologic image based risk predictor (CHIRP): identifying disease aggressiveness using sub-visual image cues from image data. Microsc Microanal. 2016 Jul;22(S3):1006-7. https://doi.org/10.1017/ S1431927616005870

45. Guillaud M, Adler-Storthz K, Malpica A, Staerkel G, Matisic J, Van Niekirk D, Cox D, Poulin N, Follen M, Macaulay C. Subvisual chromatin changes in cervical epithelium measured by texture image analysis and correlated with HPV. Gynecol Oncol. 2005;99(3 Suppl 1):S16-S23. doi:https://doi.org/10.1016/ j.ygyno.2005.07.037 PMID: 16188299

46. Cronin M, Sangli C, Liu M-L, Pho M, Dutta D, Nguyen A, Jeong J, Wu J, Langone KC, Watson D. Analytical validation of the Oncotype DX genomic diagnostic test for recurrence prognosis and therapeutic response prediction in node-negative, estrogen receptor-positive breast Cancer. Clin Chem. 2007;53(6):1084. https://doi.org/10.1373/clinchem.2006.076497.

47. Sparano JA, Paik S. Development of the 21-gene assay and its application in clinical practice and clinical trials. J Clin Oncol. 2008 Feb 10;26(5):721-8. https://doi.org/10.1200/JCO.2007.15.1068.

48. Muller D, Wolf C, Abecassis J, Millon R, Engelmann A, Bronner G, Rouyer N, Rio M-C, Eber M, Methlin G. Increased stromelysin 3 gene expression is associated with increased local invasiveness in head and neck squamous cell carcinomas. Cancer Res. 1993;53:165-9.

49. Turner BM, Skinner KA, Tang P, Jackson MC, Soukiazian N, Shayne M, Huston A, Ling M, Hicks DG. Use of modified Magee equations and histologic criteria to predict the Oncotype DX recurrence score. Mod Pathol. 2015 Jul; 28(7):921-31. https://doi.org/10.1038/modpathol.2015.50

50. Győrffy B, Karn T, Sztupinszki Z, Weltz B, Müller V, Pusztai L. Dynamic classification using case-specific training cohorts outperforms static gene expression signatures in breast cancer. Int J Cancer. 2015;136(9):2091-2098. https://doi.org/10.1002/ijc.29247 PMID: 25274406 PMCID: PMC4354298.

\section{Ready to submit your research? Choose BMC and benefit from:}

- fast, convenient online submission

- thorough peer review by experienced researchers in your field

- rapid publication on acceptance

- support for research data, including large and complex data types

- gold Open Access which fosters wider collaboration and increased citations

- maximum visibility for your research: over $100 \mathrm{M}$ website views per year

At BMC, research is always in progress.

Learn more biomedcentral.com/submissions 